\title{
X. Abkürzungsverzeichnis
}

\begin{tabular}{|c|c|c|c|}
\hline $\begin{array}{l}\text { Abk. } \\
\text { adj. }\end{array}$ & $\begin{array}{l}\text { Abkürzung } \\
\text { adjektivisch }\end{array}$ & $\begin{array}{l}\text { Feudalism. } \\
\text { Fliegerspr. }\end{array}$ & $\begin{array}{l}\text { Feudalismus } \\
\text { Fliegersprache }\end{array}$ \\
\hline Adj. & Adjektiv & Flugw. & Flugwesen \\
\hline adv. & adverbial & Forstw. & Forstwirtschaft \\
\hline Adv. & Adverb & Fotogr. & Fotografie \\
\hline Akk. & Akkusativ & Fut. & Futur \\
\hline Akk.obj. & Akkusativobjekt & Gastron. & Gastronomie \\
\hline Akt. & Aktiv & gebräuchl. & gebräuchlich \\
\hline Archäol. & Archäologie & geh. & gehoben \\
\hline Architekt. & Architektur & Gen. & Genitiv \\
\hline Art. & Artikel & geogr. & geographisch \\
\hline Astron. & Astronomie & Geogr. & Geographie \\
\hline attr. & attributiv & Geol. & Geologie \\
\hline Attr. & Attribut & Geophys. & Geophysik \\
\hline Bauw. & Bauwesen & Geschichtswiss. & Geschichtswissenschaft \\
\hline Bergm. & Bergmannssprache & Gramm. & Grammatik \\
\hline berl. & berlinisch & Handarb. & Handarbeit \\
\hline bes. & besonders & Handw. & Handwerk \\
\hline best. Art. & bestimmter Artikel & Hilfsvb. & Hilfsverb \\
\hline Bez. & Bezeichnung & Hotelw. & Hotelwesen \\
\hline bild. Kunst & bildende Kunst & Hüttenw. & Hüttenwesen \\
\hline Biol. & Biologie & Idealism. & Idealismus \\
\hline Bot. & Botanik & idealist. & idealistisch \\
\hline Böttch. & Böttcherei & Imp. & Imperativ \\
\hline BRD & Bundesrepublik Deutschland & imperial. & imperialistisch \\
\hline Buchbind. & Buchbinderei & Imperial. & Imperialismus \\
\hline Buchw. & Buchwesen & Indef.pron. & Indefinitpronomen \\
\hline bürgerl. & bürgerlich & indekl. & indeklinabel \\
\hline Chem. & Chemie & Inf. & Infinitiv \\
\hline Dat. & Dativ & Interr.pron. & Interrogativpronomen \\
\hline Datenverarb. & Datenverarbeitung & iron. & ironisch \\
\hline Dat.obj. & Dativobjekt & Jägerspr. & Jägersprache \\
\hline DDR & $\begin{array}{l}\text { Deutsche Demokratische } \\
\text { Republik }\end{array}$ & $\begin{array}{l}\text { Jh., Jhs. } \\
\text { jmd. }\end{array}$ & $\begin{array}{l}\text { Jahrhundert, Jahrhunderts } \\
\text { jemand }\end{array}$ \\
\hline Dem.pron. & Demonstrativpronomen & jmdm. & jemandem \\
\hline Eisenb. & Eisenbahn & jmdn. & jemanden \\
\hline Elektrotechn. & Elektrotechnik & jmds. & jemandes \\
\hline emot. & emotional & Jur. & Jura \\
\hline emot. neg. & emotional negativ & kap. & kapitalistisch \\
\hline emot. pos. & emotional positiv & Kap. & Kapitalismus \\
\hline etw. & etwas & Kard.zahl & Kardinalzahl \\
\hline fachspr. & fachsprachlich & Kaufm, & Kaufmannssprache \\
\hline Fem. & Femininum & Kernphys. & Kernphysik \\
\hline feudal. & feudalistisch & Kinderspr. & Kindersprache \\
\hline
\end{tabular}




\section{Abkürzungsverzeichnis}

\begin{tabular}{|c|c|c|c|}
\hline $\begin{array}{l}\text { Kochk. } \\
\text { Komp. }\end{array}$ & $\begin{array}{l}\text { Kochkunst } \\
\text { Komparativ }\end{array}$ & $\begin{array}{l}\text { Plusquamperf. } \\
\text { Pol. Ök. }\end{array}$ & $\begin{array}{l}\text { Plusquamperfekt } \\
\text { Politische Okonomie }\end{array}$ \\
\hline Konj. & Konjunktion & Poss.pron. & Possessivpronomen \\
\hline Kunstgesch. & Kunstgeschichte & präd. & prädikativ \\
\hline Kurzf. & Kurzform & präp. & präpositional \\
\hline Kurzw. & Kurzwort & Präp. & Präposition \\
\hline Kybern. & Kybernetik & Präp.obj. & Präpositionalobjekt \\
\hline landsch. & landschaftlich & Präs. & Präsens \\
\hline Landw. & Landwirtschaft & Prät. & Präteritum \\
\hline Literaturwiss. & Literaturwissenschaft & Pron. & Pronomen \\
\hline mask. & maskulin & Pronominaladv. & Pronominaladverb \\
\hline Mask. & Maskulinum & Psych. & Psychologie \\
\hline Math. & Mathematik & räuml. & räumlich \\
\hline Med. & Medizin & Refl.pron. & Reflexivpronomen \\
\hline Meteor. & Meteorologie & Rel. & Religion \\
\hline Mil. & Militär & Rel. ev. & Religion evangelisch \\
\hline Min. & Mineralogie & Rel. jüd. & Religion jüdisch \\
\hline Modalvb. & Modalverb & Rel. kath. & Religion katholisch \\
\hline monopol. & monopolistisch & Rel.pron. & Relativpronomen \\
\hline Mus. & Musik & Rundf. & Rundfunk \\
\hline nachdrückl. & nachdrücklich & s. & siehe \\
\hline $\begin{array}{l}\text { Naturwiss. } \\
\text { neutr. }\end{array}$ & $\begin{array}{l}\text { Naturwissenschaft } \\
\text { neutral }\end{array}$ & $\begin{array}{l}\text { Sammelbez. } \\
\text { scherzh. }\end{array}$ & $\begin{array}{l}\text { Sammelbezeichnung } \\
\text { scherzhaft }\end{array}$ \\
\hline Neutr. & Neutrum & Schießsp. & Schießsport \\
\hline Nom. & Nominativ & Schimpfw. & Schimpfwort \\
\hline norddt. & norddeutsch & Schreibg. & Schreibung \\
\hline Num. & Numerale & Schuhmach. & Schuhmacherhandwerk \\
\hline o. & ohne & Schülerspr. & Schülersprache \\
\hline o. ä. & oder ähnliches & schweiz. & schweizerisch \\
\hline od. & oder & Seemannsspr. & Seemannssprache \\
\hline Opt. & Optik & Sg. & Singular \\
\hline Ord.zahl & Ordinalzahl & Soldatenspr. & Soldatensprache \\
\hline örtl. & örtlich & soz. & sozialistisch \\
\hline österr. & österreichisch & Soz. & Sozialismus \\
\hline ostmdt. & ostmitteldeutsch & Soziol. & Soziologie \\
\hline Päd. & Pädagogik & spött. & spöttisch \\
\hline papierdt. & papierdeutsch & Sprachwiss. & Sprachwissenschaft \\
\hline Parl. & Parlamentarismus & Studentenspr. & Studentensprache \\
\hline Part. & Partizip & Subj. & Subjekt \\
\hline Part. Präs. & Partizip Präsens & subst. & substantivisch, substantiviert \\
\hline Part. Prät. & Partizip Präteritum & Subst. & Substantiv \\
\hline Pass. & Passiv & süddt. & süddeutsch \\
\hline Perf. & Perfekt & Superl. & Superlativ \\
\hline Pers. & Person & st. Vb. & starkes Verb \\
\hline persönl. & persönlich & sw. Vb. & schwaches Verb \\
\hline Pers.pron. & Personalpronomen & Tabakwarenind. & Tabakwarenindustrie \\
\hline Pharm. & Pharmazie & Techn. & Technik \\
\hline Philat. & Philatelie & Textil. & Textilindustrie \\
\hline Philos. & Philosophie & Transportw. & Transportwesen \\
\hline Phonet. & Phonetik & u. & und \\
\hline Phys. & Physik & u. ä. & und ähnliches \\
\hline Physiol. & Physiologie & Uhrm. & Uhrmacherhandwerk \\
\hline Pl. & Plural & umg. & umgangssprachlich \\
\hline
\end{tabular}




\section{Abkürzungsverzeichnis}

unbest. Art. unbestimmter Artikel

ungebräuchl. ungebräuchlich

unpers.

u. Z.

usw.

unpersönlich

unserer Zeitrechnung.

und so weiter

$\mathrm{Vb}$.

Verb

veralt.

verhüll.

veraltet

verhüllend

Verkehrsw. Verkehrswesen

Verlagsw. Verlagswesen

vgl.
Volksk.

vorw.

v. u. Z.

Wasserwirtsch. Wassertwirtschaft

westmdt.

Wirtsch.

westmitteldeutsch

Zahnmed. Zahnmedizin

zeitl.

Zool. Zoologie

z. T.

zum Teil 
